\title{
DSINE: Deep Structural Influence Learning via Network Embedding
}

\author{
Jianjun Wu, ${ }^{1,2}$ Ying Sha, ${ }^{1,2}$ Bo Jiang, ${ }^{1,2}$ Jianlong Tan ${ }^{1,2}$ \\ ${ }^{1}$ Institute of Information Engineering, Chinese Academy of Sciences \\ No.91 Min Zhuang Road, Haidian District, Beijing, China 100093 \\ ${ }^{2}$ School of Cyber Security, University of Chinese Academy of Sciences Beijing, China 100029 \\ \{wujianjun, shaying, jiangbo, tanjianlong\}@iie.ac.cn
}

\begin{abstract}
Structural representations of user social influence are critical for a variety of applications such as viral marketing and recommendation products. However, existing studies only focus on capturing and preserving the structure of relations, and ignore the diversity of influence relations patterns among users. To this end, we propose a deep structural influence learning model to learn social influence structure via mining rich features of each user, and fuse information from the aligned selfnetwork component for preserving global and local structure of the influence relations among users. Experiments on two real-world datasets demonstrate that the proposed model outperforms the state-of-the-art algorithms for learning rich representations in multi-label classification task.
\end{abstract}

\section{Introduction}

The use of relation information in social networks plays an important role in recommending products, promoting public opinions and detecting information anomalies. Most of existing studies focus on the homogeneous networks. These methods assume similar users are more likely to be linked together and have a closer relationship (Tang 2015). However, the relation patterns learned by these models have higher locality, resulting in a large structure overlap and a limited local structure. Meanwhile, the information sparsity is still not fully solved. To alleviate the social data sparsity problem, several effective strategies such as random walk, breadthfirst and depth-first have been proposed on heterogeneous networks. However, the above studies are hard to learn the global influence relations.

In this paper, we propose a deep structural influence learning model using network embedding, named DSINE. This model first learns the structural representations of relationships of entities to preserve first-order, second-order, and high-order proximity influence structure, and then maps these learned representations to a low-dimensional space of features that maximize the likelihood of preserving both the global and local structure of the influence relations of vertexes in embedding space.

Copyright (C) 2019, Association for the Advancement of Artificial Intelligence (www.aaai.org). All rights reserved.

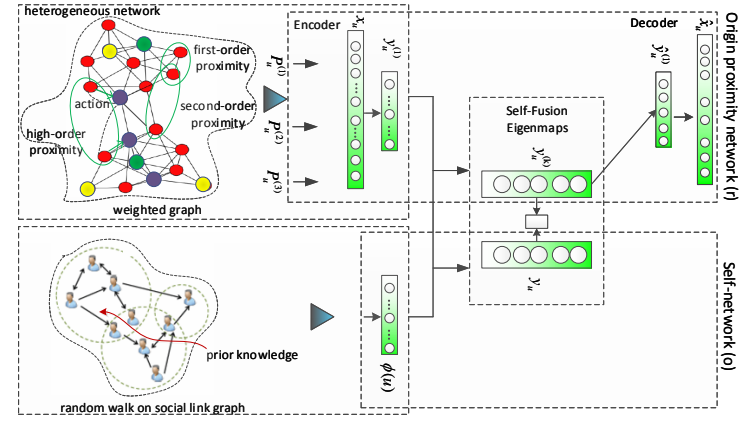

Figure 1: The framework of DSINE.

\section{The DSINE Model}

The DSINE extends the auto-encoder model via aligned self-network, as shown in Fig.1. The origin proximity network $r$ preserves structure of first-order, second-order, highorder proximity among vertexes. Likewise, self-network $o$ can learn a mapping of vertexes to a low-dimensional space of features based on random walk procedure.

In our model, we take different feature vectors extracted from first-order, second-order, high-order proximity between users as the input data of origin proximity network $r$. The term $p_{u}^{(1)}$, which is the row in first-order proximity matrix $P^{(1)}$, represents the input feature vectors for user $u$. As described in (Zhang 2017), the order proximity for each user can be independently calculated to the embedding vectors by multiple non-linear mapping functions and then map these vectors to a lower-dimensional feature space, which will combine those vectors to generate result embedding vector $y_{u}^{(k)}$ for user $u$. Meanwhile, we align origin proximity network $r$ with self-network, which learns embedding representations based on random walk guided by prior knowledge, to incorporate the embedding results into the origin proximity network to alleviate the data sparsity.

To learn different influence and preserve micro-, mesoand macro- structure between users, we design the four components to exploit first-order proximity, second-order proximity, high-order proximity and information transferring aligned self-work for maximizing the likelihood of preserving both the global and local structure of the influence 
relations of users and minimizing the reconstruction loss. Based on the first-order proximity, we can obtain a highly sparse adjacency matrix $F$. To relieve the effect of traditional auto-encoder model, we exploit the weight strategy to distinguish the non-zero features from zero features. The objective of loss function is defined as

$$
\mathcal{L}_{1 s t}=\sum_{i}\left\|\left(\hat{x}_{i}-x_{i}\right) \odot b_{i}\right\|_{2}^{2}, b_{i}=\left\{b_{i j}\right\}_{j=1}^{n}
$$

where $b_{i}$ represents $i$-th row weight vector. $b_{i j}=1$ if $f_{i j}(\epsilon$ $F)$ is 0 , otherwise $b_{i j}=k(>1)$. Similarly, the loss function of second-order proximity is represented as

$$
\mathcal{L}_{2 n d}=\sum_{i \in\left\{\psi_{i}, \cdots, \psi_{k}\right\}} \sum_{j=1}^{n}\left\|\left(\hat{x}_{i, j}^{r}-x_{i, j}^{r}\right) \odot b_{i, j}^{r}\right\|_{2}^{2}, b_{i}=\left\{b_{i j}\right\}_{j=1}^{n}
$$

where $r$ represents origin proximity network. $b_{i, j}^{r}$ is the weight vector for feature vector $x_{i, j}^{r}$. Similarly, the loss function of high-order proximity $\mathcal{L}_{h}$ is the same as Eq. (1) and Eq. (2). For origin proximity network $r$, the final loss function is defined as

$$
\mathcal{L}^{r}=\mathcal{L}_{1 s t}+\alpha \mathcal{L}_{2 n d}+\beta \mathcal{L}_{h}+\eta \mathcal{L}_{r e g}^{r}
$$

where parameters $\alpha, \beta$, and $\eta$ denote weight of loss function , $\mathcal{L}_{\text {reg }}^{r}$ is a regularizer term, which can be defined as

$$
\mathcal{L}_{r e g}^{r}=\frac{1}{2} \sum_{k=1}^{N}\left(\|\left(\hat{W}^{r(k)}\left\|_{F}^{2}+\right\|\left(W^{r(k)} \|_{F}^{2}\right)\right.\right.
$$

In addition, we use self-network $o$ for aligned origin proximity network $r$, reinforcing the embedding result in the $r$. The joint objective of minimizing loss can be represented as

$$
\mathcal{L}^{r, o}=\mathcal{L}^{r}+\varepsilon \mathcal{L}_{r, o}+\lambda \mathcal{L}_{r e g}
$$

where $\mathcal{L}_{r, o}$ denotes minimizing the loss of transforming information between $r$ and $o$, which can be defined as

$$
\mathcal{L}_{r, o}=\sum_{i=i}^{n} w_{i}\left\|\left(y_{i}^{r(k)}-y_{i}^{o(k)}\right)\right\|_{F}^{2}
$$

where $y_{i}^{r(k)}$ and $y_{i}^{o(k)}$ represents the row embedding results in network $r$ and $o$. To prevent overfitting, $\mathcal{L}_{r e g}$ is a regularizer term which is defined as

$$
\mathcal{L}_{r e g}=\frac{1}{2} \sum_{k=1}^{N}\left(\|\left(\hat{W}^{r(k)}\left\|_{F}^{2}+\right\|\left(W^{o(k)} \|_{F}^{2}\right)\right.\right.
$$

\section{Experiments}

The datasets of experiment are from Twitter dataset collected by crawler and public available RepLab-2014 ${ }^{1}$. We compare DSINE with four recently representation learning methods: Deepwalk (Perozzi 2014), LINE (Tang 2015),

\begin{tabular}{|c|c|c|c|c|c|c|c|c|}
\hline \multirow{2}{*}{ Dataset } & \multirow{2}{*}{ Metrics } & \multirow{2}{*}{ Algorithm } & \multicolumn{6}{|c|}{ Training Data } \\
\hline & & & $10 \%$ & $20 \%$ & $60 \%$ & $70 \%$ & $80 \%$ & $90 \%$ \\
\hline \multirow{10}{*}{ Twitter } & \multirow{5}{*}{ Micro-F1 } & Deepwalk & 30.79 & 32.32 & 33.80 & 32.42 & 33.83 & 34.47 \\
\hline & & LINE & 39.34 & 46.28 & 53.41 & 55.71 & 57.17 & 36.97 \\
\hline & & SDNE & 45.4 & 50.63 & 50.68 & 51.54 & 53.47 & 64.36 \\
\hline & & Node2vec & 47.67 & 45.22 & 51.81 & 53.73 & 54.29 & 59.0 \\
\hline & & DSINE & 50.00 & 50.99 & 56.57 & 64.91 & 71.05 & 73.08 \\
\hline & \multirow{5}{*}{ Macro-F1 } & Deepwalk & 44.88 & 47.96 & 46.64 & 49.25 & 47.53 & 54.18 \\
\hline & & LINE & 48.59 & 47.37 & 39.23 & 44.68 & 47.83 & 43.04 \\
\hline & & SDNE & 44.50 & 45.35 & 52.12 & 45.64 & 42.81 & 51.30 \\
\hline & & Node2vec & 44.66 & 46.48 & 54.05 & 48.64 & 53.00 & 54.12 \\
\hline & & DSINE & 52.83 & 48.43 & 62.27 & 79.03 & 79.77 & 78.52 \\
\hline \multirow{10}{*}{ RepLab-2014 } & \multirow{5}{*}{ Micro-F1 } & Deepwalk & 10.23 & 10.87 & 12.77 & 22.17 & 15.37 & 25.65 \\
\hline & & LINE & 20.47 & 20.44 & 14.09 & 13.66 & 15.39 & 15.39 \\
\hline & & SDNE & 13.64 & 18.55 & 23.09 & 22.23 & 30.75 & 41.00 \\
\hline & & Node2vec & 18.18 & 19.18 & 17.90 & 23.90 & 30.70 & 30.36 \\
\hline & & DSINE & 18.20 & 20.47 & 23.04 & 27.33 & 40.97 & 51.44 \\
\hline & \multirow{5}{*}{ Macro-F1 } & Deepwalk & $\overline{10.18}$ & 10.83 & 12.74 & 22.07 & 15.21 & 25.37 \\
\hline & & LINE & 20.32 & 20.33 & 13.97 & 13.51 & 15.16 & 15.18 \\
\hline & & SDNE & 13.53 & 18.41 & 22.85 & 22.04 & 30.48 & 40.62 \\
\hline & & Node2vec & 18.07 & 19.06 & 17.81 & 23.72 & 30.45 & 40.26 \\
\hline & & DSINE & 18.08 & 20.27 & 22.85 & 27.04 & 40.31 & 50.02 \\
\hline
\end{tabular}
SDNE (Cui 2016), Node2vec (Grover 2016). As shown in the Table 1, the results of DSINE perform better than other baseline algorithms on Twitter dataset. It demonstrates that

\footnotetext{
${ }^{1}$ http://nlp.uned.es/replab2014/
}

Table 1: Performance of multi-label classification with different training data settings on Twitter and RepLab2014.

the information of first-order, second-order, and high-order proximity can enrich the structural representation of social influence when the neighborhood of vertex is sparse in network. Similar to (Cui 2016), the performance of Deepwalk is the worst among the representation learning methods. The other methods perform relatively to be close, but our method can perform better than baselines as the size of sampled networks increases. Especially, it is more important to this task because the influence relation is more sparse.

\section{Conclusion and Future Work}

In this paper, we present a new model of structural representations learning of social influence in preserving first-order, second-order, and high-order proximity influence structure and mapping them to a low-dimensional space in heterogeneous social networks. Future extensions of our model could involve detecting community with influence structure in heterogeneous information network.

\section{Acknowledgments}

This paper is supported by National Key Research and Development Program of China under Grant No. 2017YFB0803003, No.2017YFC0820705, Natural Science Foundation of China (No.61702508).

\section{References}

Grover, A., Leskovec, J. 2016. node2vec: Scalable Feature Learning for Networks. In KDD, 855-864. ACM.

Perozzi, B., Al-Rfou, R., Skiena, S. 2014. DeepWalk: online learning of social representations. In KDD, 701-710. ACM.

Tang, J., Qu, M., Wang, M., et al. 2015. LINE: Large-scale Information Network Embedding. In WWW, 1067-1077. ACM.

Wang, D., Cui, P., Zhu W. 2016. Structural Deep Network Embedding. In KDD, 1225-1234. ACM.

Zhang, J., Xia, C., Zhang, C., et al. 2017. BL-MNE: Emerging Heterogeneous Social Network Embedding Through Broad Learning with Aligned Autoencoder. In Data Mining, 605-614. ACM. 